\title{
MODELO DEA NÃO-ARQUIMEDIANO PARA A AVALIAÇÃO DO SISTEMA DE TRANSPORTE PÚBLICO DE PASSAGEIROS EM MACAÉ
}

\author{
Anibal Alberto Vilcapoma Ignacio \\ Universidade Federal Fluminense - UFF \\ anibalvilcapoma@gmail.com \\ Léa Maria Dantas Sampaio \\ COPPE/UFRJ \\ Centro de Tecnologia - Cidade Universitária - \\ I. do Fundão - Rio de Janeiro - RJ \\ leasampaio@gmail.com
}

\begin{abstract}
Resumo
O presente artigo apresenta um método de avaliação do sistema de transporte público da cidade de Macaé, utilizando a ferramenta de análise de eficiência conhecida como DEA ou Análise Envoltória de Dados, que compara as eficiências do sistema de Macaé com os de outras cidades brasileiras. O modelo DEA aplicado é uma extensão do modelo DEA/BCC básico, que evita a ocorrência de DMU pseudo-eficientes, ou seja, um modelo DEA/BCC-Não Arquimediano que restringe os multiplicadores do modelo a um elemento infinitesimal maior do que zero. Também são apresentadas as sugestões de melhoria do sistema de transporte público de passageiros de Macaé.
\end{abstract}

Palavras chaves: DEA, DE-Não arquimediano, Sistemas de transporte.

\begin{abstract}
This paper presents an evaluation method of the public transport system of the Macae city, using the analysis tool known as DEA (Data envelopment analysis) which compares the efficiencies of Macae system with those of other cities. The DEA model used is an extension of DEA / basic BCC, which prevents the occurrence of pseudo-efficients decision makers units (DMU), ie a DEA / BCC Non-Archimedean model that restricts the multipliers to a greater than zero infinitesimal element. Suggestions for improvement of the public transport of passengers of Macaé system are also presented.
\end{abstract}

Key words: DEA, DEA- Non-Archimedean model, Transport Systems. 


\section{INTRODUÇÃO}

$\mathrm{Na}$ maioria das cidades brasileiras, o processo acelerado de urbanização não é devidamente acompanhado pela rede de serviços de transporte que, aos poucos, vai deixando de atender aos desejos e necessidades de deslocamento de grande parcela da população, inclusive ao não provir acesso universal na maioria dos casos. A ocupação desordenada e irracional do solo urbano com a existência de áreas totalmente vazias ou pouco adensadas no interior das médias e grandes cidades, ao mesmo tempo em que surgem ocupações de áreas mais distantes, faz transparecer a dissociação entre transporte e ocupação do solo e onera e dificulta o atendimento das áreas localizadas fora da mancha urbana.

Diversos mecanismos para amenizar as questões referentes ao trânsito têm sido implementados em diversos municípios do Brasil, a fim de se obter um serviço adequado, no que diz respeito ao transporte público. A queda na confiabilidade dos sistemas de transportes geram problemas ao desenvolvimento econômico das cidades e este processo, não controlado, pode levar a desregulamentação do setor. $\mathrm{O}$ elemento determinante na crise do transporte coletivo urbano é a falta de fontes de financiamento estáveis para a produção de infraestrutura adequada. A ausência de uma política de financiamento para o setor no Brasil, nos últimos 15 anos, conduziu à estagnação quase total dos investimentos. Muito pouco se produziu de corredores exclusivos, terminais de integração, abrigos adequados em paradas, apropriação de novas tecnologias. Quase nada em acessibilidade.

No que tange ao município de Macaé, o quadro acima é ainda mais grave, uma vez que este, sendo conhecido como a capital do petróleo, também é o município brasileiro que mais cresce, desde a década de 1970 até hoje. Desde então, Macaé se encontra despreparado para os impactos resultantes de intensos processos migratórios, aumentando a sua população dramaticamente em 440\%, em 36 anos, passando de 47 mil habitantes, em 1974, para 206 mil, em 2010.

Dentre os impactos que afetam Macaé, além dos ligados ao sistema de transportes, os principais são: invasões em áreas de risco e de Preservação Permanente; surgimento de favelas e de bairros periféricos sem infraestrutura; aumento das demandas por serviços públicos; aumento da violência decorrente do tráfico de drogas; aumento do custo de vida; especulação imobiliária; aumento do trânsito - média de 25 mil carros e 700 caminhões/dia, aproximadamente; demanda por infraestrutura: habitação, educação, equipamentos públicos e saneamento.

Para haver mais chances de financiamentos no setor de transportes, faz-se necessário, antes de qualquer tomada de decisão, a avaliação da eficiência de cada sistema de transporte, vigorando nas cidades e entre cidades. Tecnicamente, os problemas analisados, no artigo, são identificados no sistema de transporte de passageiros do município de Macaé e utiliza-se como ferramenta de avaliação do mesmo a Análise Envoltória de Dados (DEA). Esse é um método que busca medir a eficiência relativa de um conjunto de unidades tomadoras de decisão, chamadas de DMU (Decision Making Unit). Uma DMU é uma unidade produtiva que gera produtos utilizando insumos.

$\mathrm{O}$ artigo esta estruturado em seis seções. A primeira introduz o tema para o leitor. A segunda discute a avaliação do sistema de transporte público de Macaé, justificando o uso da ferramenta DEA. A terceira diz respeito à análise dos dados usados no modelo para a avaliação do sistema de transporte. A quarta discute sobre os modelos DEA e suas limitações. A quinta apresenta a aplicação do modelo escolhido para a avaliação proposta e, finalmente, a sexta seção apresenta as conclusões e recomendações do trabalho.

\section{O SISTEMA DE TRANSPORTE PÚBLICO DE MACAÉ}

A recente situação do sistema de transporte do município de Macaé obriga a uma análise comparativa com outros sistemas de transporte no Brasil. Este tipo de análise nos 
permite fazer um escore de eficiência de cada um dos sistemas analisados, permitindo uma comparação da eficiência de cada um deles.

As análises apresentadas a seguir têm como base o trabalho de [1].

\subsection{MEDINDO A EFICIÊNCIA E A EFICÁCIA NO TRANSPORTE PÚbLICO DE MACAÉ}

O transporte público é um serviço importante para a sociedade e deve ser garantido pelos governos, em uma sociedade organizada. Os governos devem garantir a locomoção das pessoas dos diversos níveis sociais e zelar por uma infraestrutura que permita o funcionamento do sistema atendendo as necessidades mínimas da sociedade. Contudo, segundo [1], o funcionamento de transporte público encontra-se atrelados a elevados subsídios governamentais, resultantes de custos operacionais, incorridos nas empresas prestadoras de serviços, sobretudo na cobertura de áreas com baixa densidade populacional.

Centros urbanos com alto crescimento demográfico sofrem normalmente com uma rede desordenada de transporte coletivo. Nestes casos existe a necessidade de se estruturar um sistema de transporte coordenado de forma única por um órgão gestor que promova a integração e coordenação das linhas, que estabeleça estrutura tarifária e coordene os diversos prestadores de serviço. Este órgão gestor é o principal responsável pela eficácia e eficiência do sistema. Entendendo por eficácia a qualidade de serviços ofertados e por eficiência a capacidade de atender às expectativas de viagens da população.

Os governos, no intuito de oferecer à população um sistema de transporte, lidam com interesses conflitantes e, por um lado tentam minimizar os subsídios possíveis às empresas operadoras do sistema e por outro devem exigir delas a oferta de um serviço de qualidade. Neste enfoque a redução dos subsídios pode-se dar com a busca da eficiência administrativa e operacional das contratadas.

Ramos [2] indica os principais fatores característicos de eficácia de um sistema de transporte público urbano como sendo: a acessibilidade ao sistema, determinada pela distância que os usuários têm que se deslocar da origem ao ponto de embarque; o tempo de viagem determinado pela velocidade comercial dos veículos e pela geometria das linhas; a confiabilidade medida pela incerteza que os usuários têm sobre os horários de saída e de chegada dos veículos; a freqüência de atendimento medida pela passagem consecutiva dos veículos pelos pontos de paradas; a lotação, pela característica do veiculo e a facilidade de utilização e mobilidade (este tipo de parâmetro envolve aspectos como a sinalização dos pontos de parada); a existência de abrigos nos locais de maior demanda; a divulgação de horários e mapas simplificados dos itinerários, referentes às linhas com localização dos terminais etc. Também são destacados outros fatores de eficácia, tais como: o número de passageiros transportados em relação a população, a extensão de linhas em relação à área, o nível de satisfação dos usuários etc.

Existem também indicadores de desempenho do sistema que, de forma geral, procuram minimizar os recursos utilizados, como mencionado em [1]. São exemplos destes indicadores o custo operacional por passageiro transportado; o tamanho de frota etc.

Uma análise de um sistema com um número considerável de indicadores observados isoladamente impossibilita a comparação com outros sistemas, não permitindo uma generalização dos resultados. Neste caso, o uso da ferramenta de DEA (Data Envelopment Analysis) é mais indicado. Geralmente, os sistemas de transporte podem ser vistos como unidades de decisão que utilizam insumos (inputs) para gerarem produtos (output) que são os serviços prestados. Portanto, aqui são considerados como inputs a infraestrutura, o número de veículos, a demanda, a idade média dos veículos etc. Os outputs podem ser os indicadores de eficiência e os de eficácia, tais como: os veículos/distância, o tempo de viagem etc.

Vários autores têm pesquisado a avaliação de desempenho de sistemas de transporte, como pode ser visto nos trabalhos de [3], [4], [5], [6] e [7] que apresentam estudos sobre medida de performance do sistema de ônibus americano. Lavaggi [8] aplica o DEA no sistema de transporte urbano da Itália. Husain [9] usam o DEA na avaliação do transporte publico da Malásia. Pina e Torres. Ramos et al. [10] utilizam o DEA para avaliarem a 
eficiência do setor privado e público na Espanha. [1] aplica o DEA na avaliação da eficiência dos sistemas de transporte público do Nordeste brasileiro, comparando-os com os de outras cidades do Brasil e de algumas cidades da Europa.

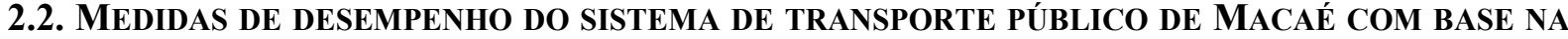 CONSTRUÇÃO DE INDICADORES}

O município de Macaé delega o serviço de transporte urbano a duas empresas particulares, porém a obrigação da prefeitura é garantir um bom transporte público à população. A prefeitura deve fiscalizar o serviço prestado e fixar o valor da tarifa, observando dois aspectos fundamentais: os cumprimentos de parâmetros operacionais preestabelecidos por ela ou pelo órgão de Gestão de Transporte; e uma tarifa que reflita a qualidade e o custo desse serviço.

Deste modo, a prefeitura precisa conhecer de forma clara os métodos de controle operacional do transporte coletivo para atuar com eficiência. Necessita também de indicadores de desempenho que sejam atualizados frequentemente para que reflitam a situação do sistema de transportes, em um horizonte de tempo. Quando a Prefeitura não acompanha estes indicadores pouco consegue, em termos de soluções que adote, uma vez que suas decisões serão realizadas de forma intuitiva focando alguns aspectos de forma isolada.

Vale lembrar que a presente análise tem por base as recomendações da Associação Nacional de Transportes Públicos - ANTP.

\subsubsection{Parâmetros de desempenho}

Os parâmetros de desempenho podem ser considerados do ponto de vista operacional e financeiro. Considerando-se os aspectos operacionais, os principais dados que sintetizam o serviço executado na operação de transporte são:

- O total diário de passageiros transportados - neste caso pode-se distinguir os possíveis passageiros gratuitos e com desconto.

- O total de quilômetros percorridos - que se subdivide em quilometragem útil e ociosa. Entende-se como quilometragem útil a soma das quilometragens da linha desde o ponto inicial ao ponto final do itinerário, ida e volta. A quilometragem ociosa se refere ao trajeto da garagem ao ponto inicial, ida e volta.

- Total diário de viagens programadas e realizadas.

- Total diário de ônibus utilizados, especificando a frota reserva e a média de veículos em manutenção.

- Cadastro dos pontos de parada e terminais.

A partir desses dados é possível se obter uma série de índices necessários ao controle da operação, permitindo-se um diagnóstico.

\subsubsection{Parâmetros de controle operacional}

Esses parâmetros são explicados nos itens abaixo.

a) Índice de passageiro por quilometro (IPK)

Esse é o índice mais importante do transporte. Ele é utilizado no cálculo da tarifa e retrata, junto com outros índices, o desempenho do serviço prestado. O IPK deve ser obtido por linha, por empresa ou pelo sistema como um todo.

IPK = número médio de passageiros / Quilometragem média rodada.

b) Índice de passageiros transportados por viagem (IPV)

É obtido a partir da relação entre o total de passageiros transportados e o número de viagens realizadas. Este retrata o desempenho da frota, de uma linha ou de uma empresa.

$\mathrm{IPV}=$ Passageiros / viagens realizadas

c) Percurso médio mensal (PMM)

Representa a seguinte relação: Total de quilômetros rodados por mês, dividido pela frota. Essa informação mostra a utilização do veículo no sistema. Quanto maior o PMM 
melhor será a utilização do veículo. A maior utilização do veículo implicará um custo quilométrico menor.

PMM = Quilometragem total / frota

d) Passageiro por viagem (PPV)

Esse dado guarda uma informação da ocupação do ônibus, ou seja, no caso em questão (Macaé) a média de carregamento de um ônibus (ao longo de um mês) é em torno de 28,3 passageiros por sentido.

$\mathrm{PPV}=$ passageiro $/ \mathrm{n}^{\circ}$ de viagens

e) Idade Média da Frota

Esse número diz respeito à qualidade do serviço prestado (conforto). A idade limite de um veículo na linha intermunicipal na Região Metropolitana é de sete anos, portanto um sistema que tem renovação de frota de mais ou menos $20 \%$ ao ano (condições normais) deve ter uma idade média máxima por volta de cinco anos. No caso de Macaé, a idade média da frota é de 2,96 anos.

\subsubsection{Dados operacionais do Sistema de Transporte Urbano do Município de Macaé}

Os dados apresentados a seguir, contêm informações operacionais por linha e por modo de transporte (ônibus e vans). Esses dados foram agrupados por tipo de ligação, a saber: troncais, alimentadoras, circulares e serranas (ver Figura 1).

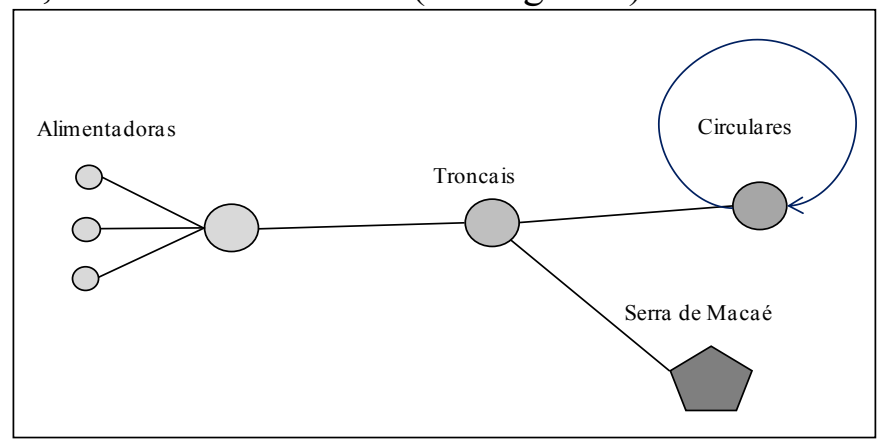

Figura 1 - dados agrupados por tipo de ligação

O objetivo desse agrupamento é analisar o carregamento por linha e por característica, para obtenção de dados que possam ser comparados com maior facilidade, determinando o carregamento de cada tipo de ligação, permitindo assim a construção de uma base de análise (ver Tabela 1).

\begin{tabular}{|l|r|r|r|r|r|r|r|r|}
\hline & $\begin{array}{c}\text { Demanda } \\
\text { mês }\end{array}$ & \multicolumn{1}{|c|}{ Km/mês } & \multicolumn{1}{|c|}{ IPK } & \multicolumn{1}{|c|}{ PMM } & $\begin{array}{c}\text { Viagens/ } \\
\text { Mês }\end{array}$ & $\begin{array}{c}\text { Passageiros/ } \\
\text { veículo }\end{array}$ & $\begin{array}{c}\text { Passageiros/ } \\
\text { Viagem }\end{array}$ & $\begin{array}{c}\text { Taxa de } \\
\text { Ocupação }\end{array}$ \\
\hline Linhas Troncais & 1.862 .614 & 443.036 & 4,20 & 6.069 & 3.719 & 797 & 56,3 & 80,41 \\
\hline Linhas Alimentadoras & 655.526 & 62.616 & 10,47 & 2.609 & 2.3 & 899 & 52,0 & 74,31 \\
\hline Alimentadoras - Vans & 680.558 & 247.261 & 2,75 & 5.261 & 4.74 & 403 & 7,5 & 29,83 \\
\hline Linhas Circulares & 175.147 & 34.789 & 5,03 & 2.046 & 1.064 & 358 & 38,4 & 54,81 \\
\hline Linhas Região Serrana & 154.08 & 172.185 & 0,89 & 13.245 & 1.152 & 334 & 31,9 & 45,64 \\
\hline Sistema & $\mathbf{3 . 5 2 7 . 9 2 4}$ & $\mathbf{9 5 9 . 8 8 8}$ & $\mathbf{3 , 6 8}$ & $\mathbf{5 . 5 1 7}$ & $\mathbf{2 4 . 8 1 6}$ & $\mathbf{6 7 6}$ & $\mathbf{2 8 , 3}$ & $\mathbf{4 0 , 3 8}$ \\
\hline Onibus & $\mathbf{2 . 8 4 7 . 3 6 7}$ & $\mathbf{7 1 2 . 6 2 7}$ & $\mathbf{4 , 0 0}$ & $\mathbf{5 . 6 1 1}$ & $\mathbf{4 8 . 9 6 8}$ & $\mathbf{7 4 7}$ & $\mathbf{5 8 , 1}$ & $\mathbf{8 3 , 0 7}$ \\
\hline Vans & $\mathbf{6 8 0 . 5 5 8}$ & $\mathbf{2 4 7 . 2 6 1}$ & $\mathbf{2 , 7 5}$ & $\mathbf{5 . 2 6 1}$ & $\mathbf{7 5 . 8 4 8}$ & $\mathbf{4 8 3}$ & $\mathbf{9 , 0}$ & $\mathbf{3 5 , 8 9}$ \\
\hline
\end{tabular}

Tabela 1 - agrupamento de dados para análise

Tendo-se como base as informações acima, o carregamento de algumas linhas troncais se encontra com um índice alto, quando comparado ao padrão adotado pelo DETRORJ que é de 42,35 passageiros por viagem.

Outra maneira de se analisar os dados é adotando-se uma ocupação física de 70 passageiros por veículo. Através deste método, se encontra em uma mesma ligação, um índice de $98,48 \%$ de taxa de ocupação, muito perto da saturação da capacidade. Analisando-se os 
dados, observa-se que o sistema troncal apresenta um índice de carregamento físico de $80,41 \%$, que é traduzido em baixo conforto para os usuários.

Considerando-se o sistema de alimentação, o carregamento também é alto. Os números encontrados passam em muito do parâmetro adotado pelo DETRO, ou seja, 74,31\% em média para as Linhas Alimentadoras. Em Macaé, a ocupação física chega a ser de 94,20\% na linha A-61 - Bairro Lagomar X T. Lagomar. Nas Linhas Circulares essas ocupações se apresentam com índices mais baixos ao padrão adotado pelo DETRO. Nas Linhas Circulares, a ocupação física foi calculada em 54,81\%, mas esse tipo de ligação costuma ter um IPK alto, pois os trajetos tendem a ser mais curtos e o sobe-e-desce tende a ser maior.

As ligações para a Região Serrana do Município apresentam taxas de ocupação com média de 45,64\%, ao longo do mês. Como se tratam de linhas com extensões maiores o IPK tende a ser baixo, como já era de se esperar. A ligação S13-T - Central X Frade chega a ter 1,02 de IPK. Este tipo de linha tem característica pendular, geralmente são ligações com pouca rentabilidade operacional. A adoção do sistema de tarifa única é punitiva para as linhas de grande extensão conjugadas a um baixo IPK. O equilíbrio econômico financeiro destas linhas mostra-se difícil de ser conseguido. Geralmente, após a implantação de sistemas de tarifa única, a qualidade do serviço costuma cair. Este fenômeno pode ser observado na Zona Oeste da Cidade do Rio de Janeiro. Com a queda da qualidade do serviço, cresce a o grau de insatisfação dos usuários, apesar da queda das tarifas.

Nas alimentadoras operadas por veículos do tipo Vans, a ocupação tem outro padrão, pois a capacidade veicular adotada para o estudo é de 25 passageiros por veículo. Tendo como esse o parâmetro, os dados da empresa municipal Macaé Transito e Transporte (MACTRAN) revelam uma ocupação de $29,83 \%$, ou seja, 7,45 passageiros por viagem e $1 / 3$ de ocupação veicular, demonstrando uma melhor utilização das viagens, no ponto de vista do usuário. Para o Sistema de Transporte Público de Macaé estes números não são bons, uma vez que revelam uma oferta acima da demanda com impactos óbvios no cálculo tarifário.

\section{ANÁLISE DOS DADOS CONSIDERADOS NA AVALIAÇÃO DO SISTEMA}

No presente trabalho, dada a disponibilidade dos dados e os critérios, foram considerados nove sistemas de transporte das seguintes cidades: Macaé (RJ), São Gonçalo (RJ), Volta Redonda (RJ), Araraquara (SP), Botucatu (SP), Piracicaba (SP), Juiz de Fora (MG), Vitória (ES) e Teresina (PI). Para se ter uma ideia das características socioeconômicas das cidades que servem como base de comparação, são mostrados a seguir os dados sobre a evolução da população e do PIB entre 1999 e 2004, das mesmas.

A Figura 2 mostra o crescimento da população, entre 1999 e 2004, de cada uma das cidades analisadas. A cidade que teve maior crescimento neste período foi Macaé com um percentual de aproximadamente $17 \%$ e em seguida a cidade de Araraquara com 9\% de crescimento. Um aspecto importante a ser mostrado é que, considerando-se o ano de 1999, as taxas de crescimento da população estão decrescendo.

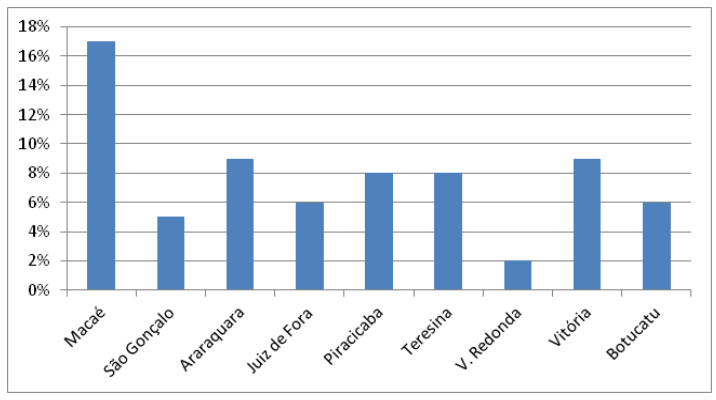

Figura 2 - Taxas de crescimento da população

Os dados disponíveis para se avaliar o sistema de transporte de cada uma das cidades são mostrados na Tabela 2 . 


\begin{tabular}{|c|r|r|r|r|r|r|r|r|r|}
\hline & Macaé & S. Gonçalo & Araraquara & Juiz de Fora & Piracicaba & Teresina & V.Redonda & Vitória & Botucatu \\
\hline $\mathrm{N}^{\mathrm{o}}$ de linhas & 38 & 69 & 24 & 231 & 92 & 89 & 44 & 29 & 40 \\
\hline Viagens & 124.816 & 108.333 & 62.774 & 373.230 & 142.324 & 131.274 & 86.730 & 42.481 & 30.000 \\
\hline Demanda & 3.527 .924 & 4.580 .000 & 1.381 .257 & 9.953 .640 & 2.581 .077 & 7.493 .033 & 3.600 .242 & 2.558 .377 & 583.333 \\
\hline $\mathrm{PMM}$ & $5.516,6$ & $6.670,3$ & $6.168,7$ & $7.088,2$ & $6.261,4$ & $6.385,3$ & $8.051,5$ & $6.745,3$ & $5.833,3$ \\
\hline $\mathrm{Km}$ & $959.887,9$ & $3.041 .666,7$ & $573.690,8$ & $3.600 .815,9$ & $1.346 .199,3$ & $3.722 .658,1$ & $1.666 .666,7$ & $694.766,0$ & $291.666,7$ \\
\hline $\begin{array}{c}\text { Km das } \\
\text { linhas }\end{array}$ & 825,9 & 960,0 & 564,5 & $3.410,0$ & $1.566,0$ & 282,8 & $1.050,0$ & 710,0 & 400,0 \\
\hline FROTA & 174 & 456 & 93 & 508 & 215 & 583 & 207 & 103 & 50 \\
\hline $\begin{array}{c}\text { Passageiros/ } \\
\text { viagem }\end{array}$ & 28,3 & 42,3 & 22,0 & 26,7 & 18,1 & 57,1 & 41,5 & 60,2 & 19,4 \\
\hline $\begin{array}{c}\text { Pass. Por } \\
\text { Onibus/dia }\end{array}$ & 747,3 & 334,8 & 495,1 & 653,1 & 400,2 & 428,4 & 579,7 & 828,0 & 388,9 \\
\hline IPK & 3,68 & 1,51 & 2,41 & 2,76 & 1,92 & 2,01 & 2,16 & 3,68 & 2,00 \\
\hline Idade Média & 2,96 & 3,70 & 7,50 & 4,30 & 5,40 & 4,20 & 4,90 & 6,30 & 1,00 \\
\hline $\begin{array}{c}\text { Vel. média } \\
\text { km/h }\end{array}$ & 12 & 50 & 40 & 17 & 18 & 22 & 22 & 30 & 40 \\
\hline
\end{tabular}

Tabela 2 - Dados disponíveis para avaliar a eficiência dos sistemas de transporte.

\section{DISCUSSÃO SOBRE OS MODELOS DEA E SUAS LIMITAÇÕES}

De acordo com o trabalho de [11], uma das mais importantes inovações introduzidas pela DEA, desenvolvida por [12], foi a habilidade de contornar a rigidez de um índice de eficiência, no qual unidades decisoras (DMU) em análise são atribuídas a um conjunto de pesos sobre variáveis (inputs e outputs), que são constantes em todas as unidades e determinadas por uma decisor (DM).

\subsection{POSSIBILIDADES DE INVIABILIDAdE NOS MODELOS DEA}

Uma das limitações mais severas dos modelos convencionais DEA é a excessiva flexibilidade de seus pesos, o que permite a busca da mÁxima eficiência da DMU, através da seleção de uma combinação de pesos que: ou é plausível porque ignora uma ou mais variáveis, ou é inaceitável, pois é incompatível com o julgamento de analistas a serviço do decisor (ver [11]).

A necessidade de um equilíbrio entre a rigidez e a flexibilidade excessiva tem levado ao desenvolvimento de modelos DEA com restrições aos pesos. Estes modelos restringem os pesos em todas as DMU. A liberdade de escolha é restrita mas, nao eliminada. Diversos métodos podem impor restrições, tais como os identificados e classificados por [13] e [14].

Uma primeira abordagem se refere à restrição dos multiplicadores que pode impor limites superior e inferior a estes e foi proposta por [15] e generalizada por [16]. Tal método tambem pode limitar um raio de multiplicadores, tal como o método da região de segurança, desenvolvido por [17] e o método de cone ratio, desenvolvido por [18] e [19] e usado por [20]. A desvantagem desta abordagem, enfatizada no trabalho de [21], é que as variáveis e seus multiplicadores associados podem ter unidades de medida e níveis de magnitude muito diferentes, o que complica a fixação de limites significativos que não provoquem inviabilidade no programa dos multiplicadores do modelo DEA.

A segunda abordagem consiste em restringir os inputs virtuais (ou outputs virtuais ou ambos), definidos como o produto de um multiplicador por uma variável correspondente. Este método foi desenvolvido por [22], com o objetivo de impor limites superior e/ou inferior sobre quotas de inputs virtuais (ou outputs), do total dos inputs ou outputs virtuais do modelo; e por [14] com o objetivo de impor limites sobre proporções (ratios) de inputs virtuais (ou outputs virtuais).

Uma vantagem desta abordagem é que as variáveis virtuais são independentes das unidades de medida e da ordem e magnitude das variáveis, facilitando a interação entre o decisor e o analista de DEA. A desvantagem é o grande número de restrições que devem ser impostas ao programa dos multiplicadores, o que aumenta a probabilidade de inviabilidade.

Deste modo, a inviabilidade é um problema potencial com as duas abordagens para a imposição de restrições aos pesos, embora por razões diferentes. De fato, a inviabilidade 
ocorre com freqüência em ambas as abordagens e não é facilmente prevista pelo DM ou pelo analista DEA.

\section{2. 'PSEUDO-EFICIÊNCIA' NOS MODELOS DEA}

Como já discutido, a prática da aplicação de modelos DEA tem demonstrado que, em alguns casos, os modelos clássicos mostram certas distorções. Isto significa que, se um dos multiplicadores v(i) ou u(r), atribuídos a uma particular DMU "eficiente" for igual a zero, então esta DMU na verdade é "pseudo-eficiente", já que faz parte de uma das faces paralelas da fronteira, a um dos eixos das coordenadas (x ou y), nos modelos BCC (ver Figura 3).

Visualiza-se este fato quando, aumentando-se ou diminuindo-se o valor do input correspondente (ou o output correspondente) ao peso que vale zero, o output (ou input) associado a ele fica sempre inalterado.

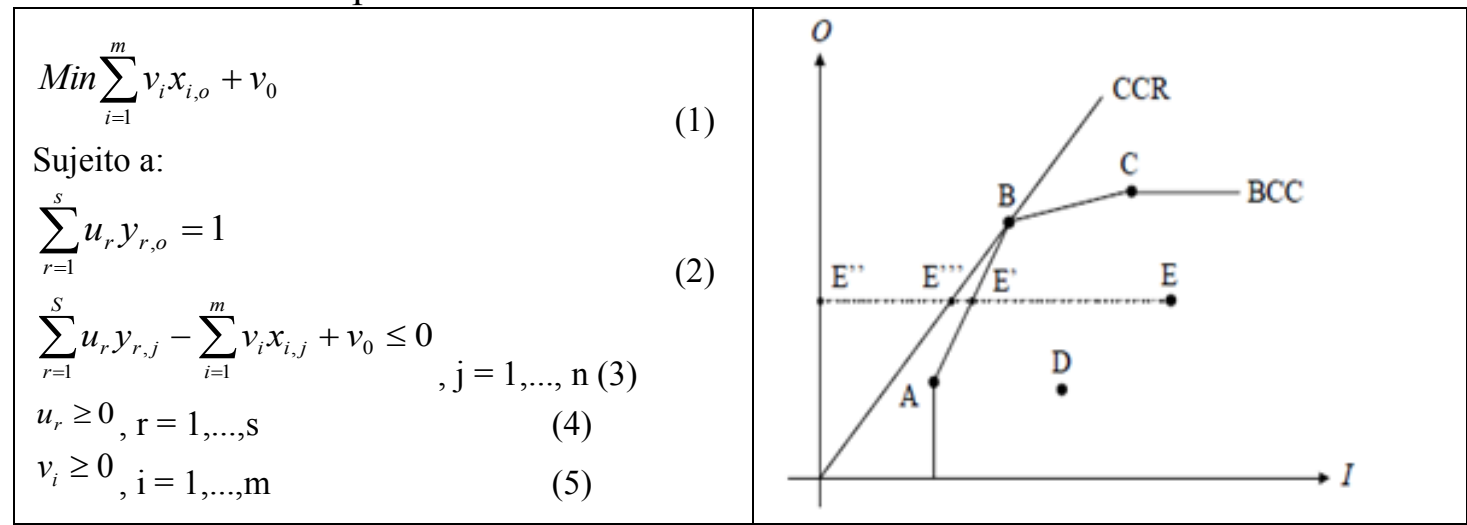

Figura 3 - Fronteiras DEA BCC/CCR (GOMES JR. e SOARES DE MELLO, 2007)

$\mathrm{Na}$ função-objetivo (1) do modelo BCC_MO se quer minimizar os insumos ou input virtual mais a variável de escala v0. A restrição (2) fixa o produto ou output virtual em uma constante igual a um. A restrição (3) estabelece que a soma do produto ou output virtual mais a variável de escala v0 deve ser menor ou igual ao insumo ou input virtual. O modelo dual, associado a este, é o 'modelo do envelope' BCC_EO, orientado a output ou produto.

No presente trabalho o modelo usado é uma extensão do modelo BCC básico, baseado no trabalho de [23], para se evitar a ocorrência de DMU "pseudo-eficientes", isto é, trata-se de um modelo BCC-Não Arquimediano (BCCNA) que restringe os multiplicadores a um elemento infinitesimal maior do que zero e, com isso, evitar tambem a ameaça de inviabilidade. Portanto, a modificação do modelo básico é a substituição das restrições (4) e (5) pelas (6) e (7), dadas abaixo:

$$
\begin{aligned}
& u_{r} \geq \varepsilon, \mathrm{r}=1 \ldots \mathrm{s} \\
& v_{i} \geq \varepsilon, \mathrm{i}=1 \ldots \mathrm{m} .
\end{aligned}
$$

Entretanto, só esta modificação ainda não garante a confiabilidade do modelo escolhido, pois, a solução de qualquer modelo DEA que envolve elemento não-arquimediano requer a escolha de um adequado intervalo para o valor deste $\varepsilon$.

De acordo com [24], o papel do valor apropriado do $\varepsilon$-infinitesimal não-arquimediano nos modelos de DEA, é desempenhado quando os programas lineares associados a estes se mostram inviáveis, na forma dos multiplicadores, e ilimitados, na forma do envelope, para certos valores de $\varepsilon$. Neste trabalho, os autores mostram que o limite superior para o $\varepsilon$, proposto por [25], a fim de garantir a viabilidade da forma de multiplicadores e a limitação na forma de envelope dos modelos CCR e BCC é inválido. [24] definem um intervalo de segurança por meio de um simples programa linear aplicado. Um valor de segurança do $\varepsilon$ é determinado e aplicado no mesmo exemplo empírico usado por [25].

Jahanshahloo e Khodabakhshi [23] determinam um intervalo de segurança para o elemento $\varepsilon$, usando um modelo que melhora, tanto os outputs na sua forma dual, quanto viabiliza o seu modelo dos multiplicadores, mediante o teorema da dualidade. Também é 
exibido um exemplo ilustrativo, no qual o valor apropriado deste elemento satisfaz um intervalo de segurança $\left(0, \varepsilon^{*}\right)$, dado pela expressão:

$\varepsilon^{*} \leq\left\{1 / \operatorname{Maxj}\left[\sum \mathrm{r} y(\mathrm{r}, \mathrm{j})\right]\right\}$.

Os modelos de DEA escolhidos foram implementados na ferramenta de modelagem AIMMS (Advanced integrated multidimensional modeling software) que, segundo [26], tratase de um ambiente computacional de desenvolvimento abrangente em modelagem de programação matemática, no qual podem ser criadas plenamente aplicações funcionais, prontas para serem usadas por pessoas mais leigas ou usuários finais.

\section{APLICAÇAO DO MODELO E DISCUSSÃO DOS RESULTADOS}

As cidades ou DMU são avaliadas em cada modelo DEA proposto na metodologia, partindo do princípio de que apresentam diferentes desempenhos, processando os mesmos tipos de insumos para darem origem ao mesmo tipo de produto.

Assim as variáveis escolhidas para o modelo são:

\begin{tabular}{|l|l|}
\hline Inputs - Insumos & Ouputs - Produto \\
\hline X1: Demanda & Y1: PMM \\
\hline X2: Frota & Y2: $1 /$ IPK \\
\hline X3: Idade da Frota & Y3: Velocidade média da frota \\
\hline
\end{tabular}

O modelo usado para rodar o DEA é o BCC orientado a Output, o qual busca maximizar o nível de outputs, mantendo o nível de inputs observado, como pode ser visto na seção 4. A ferramenta usada para se implementar e rodar o modelo é o AIMMS [26] Os resultados são mostrados na Tabela 3 , abaixo.

\begin{tabular}{|l|r|r|}
\hline \multicolumn{1}{|c|}{ Município } & $\begin{array}{c}\text { DEA BCC orientado a } \\
\text { Ouput (\%) }\end{array}$ & $\begin{array}{c}\text { DEA não } \\
\text { Arquimediano/BCC } \\
\text { orientado a Ouput \% }\end{array}$ \\
\hline Macaé & 79 & 62 \\
\hline São Gonçalo & 100 & 85 \\
\hline Araraquara & 100 & 93 \\
\hline Juiz de Fora & 92 & 38 \\
\hline Piracicaba & 96 & 93 \\
\hline Teresina & 88 & 52 \\
\hline Volta Redonda & 100 & 84 \\
\hline Vitória & 100 & 78 \\
\hline Botucatu & 100 & $\mathbf{1 0 0}$ \\
\hline
\end{tabular}

Tabela 3 - Resultados do modelo DEA BCC orientado a Ouput e DEA não Arquimediano/BCC orientado a Ouput

Os resultados das análises apontam apenas a cidade de Botucatu (SP) como sendo eficiente em termos de sistema de transporte público, nos dois modelos DEA. Pode-se afirmar que, através dos resultados que o município de Macaé apresenta desempenhos muito ruins (79\% e 62\%), em relação aos municípios considerados neste estudo. Em outras palavras, para que o município de Macaé possa atingir a fronteira de eficiência alcançada por São Gonçalo, Volta Redonda, Vitória, Araraquara e Botucatu, o Sistema de Transporte de Macaé precisa melhorar a sua performance em $26,58 \%$ e no segundo modelo faltaria $38 \%$, em relação à única cidade com sistema de transporte público eficiente.

O sistema de transporte de Macaé como está não é eficaz nem eficiente e, portanto sugere-se o redesenho do mesmo e a avaliação de cada elemento que o compõe, detalhadamente.

A título de comparação pode-se mencionar Volta Redonda, que está na fronteira da eficiência do modelo DEA arquimediano e com $84 \%$ de escore de eficiência no modelo não arquimediano, possui uma eficiência relativa maior do que a de Macaé (62\%) e uma demanda por transportes semelhante à de Macaé, porém operando com 33 carros a mais $(18,96 \%$ da atual frota do Município de Macaé). Já a velocidade operacional observada no sistema de 
Volta Redonda é 83,3\% maior em relação à praticada em Macaé. Portanto, a combinação de uma frota menor com uma velocidade muito menor explica, em parte, a falta de eficiência de Macaé, em comparação ao sistema de Volta Redonda.

Estima-se em 2,57 segundos o tempo de desembarque por passageiro. Aplicando-se a mesma taxa para o embarque, temos 5,14 segundos perdidos para cada ciclo de embarque e desembarque por passageiro. Como cada carro em Macaé carrega, ao longo do dia, 174 passageiros a mais por carro, em relação ao carregamento, observado na cidade de Volta Redonda, pode-se afirmar que, em média, cada carro é penalizado (parado) em 15 minutos por dia em Macaé. Para a frota inteira que circula em Macaé, a penalização atinge a marca de 43,5 horas por dia. Só na operação de embarque, o sistema implantado em Macaé perde $1,4 \%$ de tempo na operação em relação à Volta Redonda.

Tomando-se como base apenas um dos parâmetros mais sensíveis do sistema com o objetivo de ilustrar essa seção, a operação atual do Transporte Público de Macaé desperdiça recursos - talvez o mais sensível deles - o tempo das pessoas - uma vez que as velocidades operacionais são muito baixas. Uma hora desperdiçada em engarrafamentos ao longo de 35 anos de trabalho equivale a um ano inteiro de um trabalhador produtivo jogado fora.

Em média, a Velocidade das Linhas Alimentadoras está na casa dos $12 \mathrm{~km} / \mathrm{h}$, a das Linhas Circulares atinge $13 \mathrm{~km} / \mathrm{h}$ e a das Linhas Troncais eleva-se a $18 \mathrm{~km} / \mathrm{h}$, mesmo assim, para as Linhas Troncais T12, T22, T41 e T51 a velocidade média situa-se ao redor dos 12 $\mathrm{km} / \mathrm{h}$. Este quadro, de velocidades operacionais muito baixas implica na elevação da ineficiência do sistema. A título de comparação, a velocidade média numa ciclovia é equivalente a $16 \mathrm{~km} / \mathrm{h}$.

Como as velocidades operacionais são muito baixas e o número de passageiros transportados por dia por ônibus é alto (747 passageiros/ônibus/dia), a ocupação dos carros está sempre muito próxima do limite de capacidade. Assim, na percepção dos usuários do sistema, os ônibus circulam lentamente e permanentemente cheios. Tal cenário é agravado quando ocorrem os horários de pico. Viagens demoradas em ônibus lotados atingem diretamente a qualidade de vida da cidade. Para que o Sistema de Transporte Público de Macaé atinja um padrão considerado ótimo, as velocidades médias teriam que ser duplicadas. Macaé está muito abaixo dos padrões de eficiência aceitáveis. É urgente a reestruturação do sistema.

A mancha urbana de Macaé espraia-se ao longo da linha do litoral. As áreas urbanizadas mais distantes situam-se a menos de $5 \mathrm{~km}$ da linha de costa. Por outro lado, a partir da rodoviária, no sentido NE chega-se a franja urbana do município, já em Cabiúnas, em menos de $10 \mathrm{~km}$. No sentido $\mathrm{SW}$, tendo a rodoviária como ponto central, atinge-se o Parque dos Tubos, já na divisa com o Município de Rio das Ostras, em menos de $10 \mathrm{~km}$, também. Em função das dimensões urbanas de Macaé o tratamento dispensado ao equacionamento do transporte urbano parece ser inadequado.

Numa primeira aproximação, a construção de cinco terminais de integração e transferência numa região tão exígua, sugere um tratamento fora das boas práticas de transporte para cidades médias. Muitos terminais de transbordo e transferência numa região pouco adensada e relativamente pequena implicam no aumento da impedância do sistema de transporte coletivo. Também não existem diferenças significativas de capacidade entre os veículos que operam as linhas troncais e as linhas alimentadoras, portanto, trata-se de um sistema, que em última análise, penaliza o usuário. No transbordo de uma linha alimentadora para uma linha troncal não existem ganhos de velocidade e capacidade para o sistema, portanto, tal concepção mostra-se inútil para o sistema de transporte público de Macaé.

A partir dos dados utilizados nas modelagens DEA, a fronteira eficiente de velocidade operacional do sistema baseado em ônibus está ao redor dos $40 \mathrm{~km} / \mathrm{h}$. Em sistemas não ótimos, a velocidade está entre 20 e $30 \mathrm{~km} / \mathrm{h}$, como mostrado na Tabela 3 . Em Macaé, uma viagem do Parque dos Tubos a área central da cidade (rodoviária) leva cerca de uma hora. 


\section{CONCLUSÕES E RECOMENDAÇÕES}

Diante da discussão dos resultados exposta acima, conclui-se que, para se reduzir a ineficiência do Sistema de Transporte Público de Macaé, várias frentes de trabalho são necessárias, sobretudo em relação às velocidades operacionais. Podem-se citar recomendações para ações de melhoria do sistema como a segregação de tráfego com faixas ou pistas exclusivas, preferencialmente junto ao canteiro central (se a via permitir), priorizando-se o ônibus, em relação aos demais veículos; também a reprogramação semafórica da cidade com a implantação de sistemas de controle de tráfego seria interessante, além do reordenamento de tráfego, através de corredores exclusivos para ônibus, na área central da cidade, com redistribuição de pontos de paradas. Poderia haver algumas restrições, tais como: de estacionamentos de carga e descarga e de veículos de passeio, na zona central da cidade, bem como da circulação de veículos lentos, no perímetro desta mesma área.

A construção de vias, visando o aumento da capacidade de tráfego, nos pontos de estrangulamento, poderia ser uma boa estratégia para os seguintes pontos:

- Rodovia Amaral Peixoto, entre o Parque dos Tubos e o Centro da cidade, duplicando a existente;

- Entre a zona central da cidade e os bairros que se situam ao Norte do Rio Macaé, melhorar a circulação de veículos, construindo uma nova ponte sobre o rio Macaé.

$\mathrm{Na}$ Linha Verde, pode ser interessante para interligar melhor entre si, o tecido urbano da cidade e a transferência de equipamentos urbanos para a franja urbana da cidade notadamente a Rodoviária Intermunicipal. Isto também melhoraria o sistema de embarque e desembarque de passageiros, ao longo das linhas com a construção de BRT, quando aplicável.

Outro ponto importante que pode ser recomendado é o redesenho do sistema de linhas, a fim de tornar as viagens mais rápidas, diretas e eficientes. Com as linhas redesenhadas torna-se mais fácil o redimensionamento das frotas e a redistribuição dos headways.

Por fim, a introdução de um sistema automatizado de arrecadação tarifária para o transporte urbano, com bilhetagem eletrônica, simplificaria a movimentação ainda mais depois da retirada das lombadas e redutores de velocidade, ao longo das vias principais.

\section{REFERÊNCIAS BIBLIOGRÁFICAS}

[1] Peres, W.; Muros, S.; Ignacio, A.A.V.; Oliveira, A.; Peres, P. "Avaliação do desempenho e cálculo da eficiência do sistema de transporte urbano de passageiros com base na Análise Envoltória de Dados - DEA”. Relatório técnico da FUNEMAC, 2007.

[2] Ramos, S.B, Sampaio Y., Sampaio, L.M.B. 2006. "Eficiência do Sistema de Transporte Público no Nordeste com Análise Envoltória de Dados(DEA)", Revista Econômica do Nordeste, V 37, no 2, pp 261.

[3] Ramos, R.E.B. Uma Estratégia de Política Regulatória para Competiçao e Qualidade no Transporte Público Urbano Brasileiro. In: Transporte em Tempos de Reforma. SANTOS, E.; ARAGÃO, J.; LGE. Brasília, 2000.

[4] Fielding, G.J, "Managing Transit Strategically". São Francisco, C.A. Jossey-Bass, 1987.

[5] Karlaftis, M.G,"A DEA approach for evaluating the efficiency and effectiveness of urban transit systems", European Journal of Operational Research, V. 152, pp 354-364, 2004.

[6] Viton, P.A, "Changes in multi-model bus transit efficiency 1988-1992", Transportation, v.25 pp. 1-21, 1998.

[7] Chu, X., Fielding, G.J.,Lamar, B.W.,1992, "Measuring transit performance using data envelonpment analysis", Transportation Research, v 26A, n.3, pp 223-230.

[8] Nolan, J.F., 1996," Determinants of productive efficiency in urban transit", Logistic and Transportation Review, v.32,n.3, pp 319-342.

[9] Levaggi, R. Parametric and nonparametric approach to efficiency: the case of urban transport in Italy. StudiEconomici, v. 49, n. 53, p. 67-88, 1994. 
[10] Husain, N. Abdullah, M., Kuman, S.," Evaluating public sector efficiency with data envelopment analysis (DEA): a case study in road transport department.Selangor, Malaysia”. Total Quality Management, v. 11, n. 4-6, p. S830-S836, 2000.

[11] Pina, V.; Torres, L, Analysis of the efficiency of local government services delivery: an application to urban public transport. Transportation Research, Part A, v.35, p. 929-944, 2001.

[12] Lins, M.P.E., Silva, A.C.M., Lovell, C.A.K. "Avoiding infeasibility in DEA models with weight restrictions". European Journal of Operational Research 181, pp.956-966 (2007).

[13] Charnes, A., Cooper, W.W. E Rhodes, E. 1978, "Measuring the efficiency of decisionmaking units", European Journal of Operational Research, Vol.: 2, 429-444.

[14] Allen, R., Athanassopoulos, A., Dyson, R.G., Thanassoulis, E., "Weights restrictions and value judgements in data envelopment analysis: Evolution, development and future directions". Annals of Operations Research 73, 13-34, 1997.

[15] Pedraja-Chaparro, R., Salinas-Jimenez, J., Smith, P. "On the role of weight restrictions in data envelopment analysis". Journal of Productivity Analysis 8 (2), 215-230, 1997.

[16] Dyson, R.G., Thanassoulis, E. "Reducing weight flexibility in data envelopment analysis". Journal of the Operational Research Society 39 (6), 563-576, 1988.

[17] ROLL, Y., COOK, W., GOLANY, B. "Controlling factor weights in data envelopment analysis". IIE Transactions, 23 (1), 2-9, 1991.

[18] Thompson, R.G.; Langemeier, L.N.; Lee, C.; Lee, E. \& Thrall, R. (1990). The Role of Multiplier Bounds in Efficiency Analysis with Application to Kansas Farming. Journal of Econometrics, 46, 93-108.

[19] Charnes, A.; Cooper, W.; Wei, Q.L. \& Huang, Z.M. (1989). Cone Ratio Data Envelopment Analysis and Multiple Objective Linear Programming. International Journal of Management Science, 20(7), 1099-1118.

[20] Charnes, A., Cooper, W.W., Huang, Z.M., Sun, D.B. "Polyhedral cone-ratio DEA models with an illustrative application to large commercial banks". Journal of Econometrics 46 (1-2), 73-91, 1990.

[21] Kornbluth, J.S.H. "Analysing policy effectiveness using cone restricted data envelopment analysis". Journal of the Operational Research Society 42 (12), 10971104, 1991.

[22] Sarrico CS e Dyson RG. Restricting virtual weights in data envelopment analysis. European Journal of Operational Research 159: 1, 17-34, 2004.

[23] Wong, Y.-H.B., Beasley, J.E. "Restricting weight flexibility in data envelopment analysis". Journal of the Operational Research Society 41 (9), 829-835, 1990.

[24] Jahanshahloo, G. R.; Khodabakhshi, M., "Determining assurance interval for nonArchimedean element in the improving outputs model in DEA", Applied Mathematics and Computation, vol. 151, pp. 501-506, 2004.

[25] Mehrabian, S., Jahanshahloo, G. R., Alirezaee, M. R., Amin, G. R., “An assurance interval for the non-Archimedean epsilon in DEA models" Operations Research, 48, 2; ABI/INFORM Global, pg. 344, 2000.

[26] Ali, A. I. E Seiford, L. M., "Computational accuracy and infinitesimals in Data envelopment analysis", Infor, vol. 31, n. 4, pp. 290-297, 1993.

[27] Ignacio, A.A.V.; Ferreira Filho, V.J.M. O Uso de Software de Modelagem AIMMS na Solução de Problemas de Programação Matemática. Pesquisa Operacional, Rio de janeiro, Rio de Janeiro, v. 24, n. 1, p. 197-210, 2004. 